
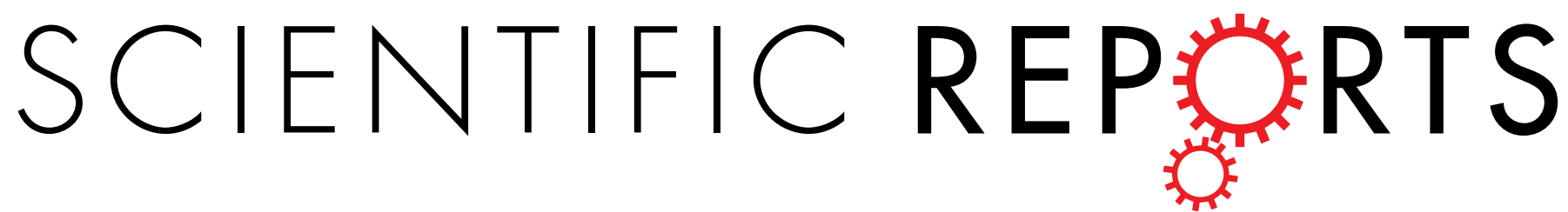

\title{
OPEN Automation of human pluripotent stem cell differentiation toward retinal pigment epithelial cells for large-scale productions
}

Received: 10 April 2019

Accepted: 5 July 2019

Published online: 23 July 2019
Florian Regent ${ }^{1,2}$, Lise Morizur ${ }^{1,2,3}$, Léa Lesueur ${ }^{1,2,3}$, Walter Habeler ${ }^{1,2,3}$
Alexandra Plancheron
$1,2,3$
, Karim Ben M'Barek $\mathbb{D}^{1,2,3}$ \& Christelle Monville

Dysfunction or death of retinal pigment epithelial (RPE) cells is involved in some forms of Retinitis Pigmentosa and in age-related macular degeneration (AMD). Since there is no cure for most patients affected by these diseases, the transplantation of RPE cells derived from human pluripotent stem cells (hPSCs) represents an attractive therapeutic alternative. First attempts to transplant hPSC-RPE cells in AMD and Stargardt patients demonstrated the safety and suggested the potential efficacy of this strategy. However, it also highlighted the need to upscale the production of the cells to be grafted in order to treat the millions of potential patients. Automated cell culture systems are necessary to change the scale of cell production. In the present study, we developed a protocol amenable for automation that combines in a sequential manner Nicotinamide, Activin A and CHIR99021 to direct the differentiation of hPSCs into RPE cells. This novel differentiation protocol associated with the use of cell culture robots open new possibilities for the production of large batches of hPSC-RPE cells while maintaining a high cell purity and functionality. Such methodology of cell culture automation could therefore be applied to various differentiation processes in order to generate the material suitable for cell therapy.

Human pluripotent stem cells (hPSCs), including human embryonic stem cells (hESCs) and human induced pluripotent stem cells (hiPSCs) are characterized by unlimited self-renewal and their ability to differentiate into any cell type. Due to these properties, extensive efforts have been done to use them as a source material for cell therapy to repair damaged tissues. At the forefront of cell therapy, the replacement of retinal pigment epithelium (RPE), the monolayer of pigmented cells localized between the neural retina and the choroid, acts as a proof of concept. RPE cells play crucial roles in sight and their dysfunction or their loss may engender the secondary loss of photoreceptors ${ }^{1}$. RPE cells are altered in 5-6\% of Retinitis Pigmentosa cases (RP, a group of rare hereditary diseases) and in Age-related Macular Degeneration (AMD) ${ }^{2,3}$. AMD is the leading cause of blindness in developed countries with more than 150 million people affected worldwide, a figure that will increase in the coming years ${ }^{4}$. It can be classified into two groups, dry (atrophic) or wet (exudative), which is based on the presence of a choroidal neovascularization. While the understanding of the molecular mechanisms underlying wet AMD, which accounts for approximately $10-15 \%$ of AMD patients, led to the development of effective anti-VEGF drugs, there is still no treatment for dry AMD and for most of $\mathrm{RPs}^{5,6}$. As such, the transplantation of RPE cells derived from human pluripotent stem cells (hPSC-RPE) represents an attractive strategy for treating retinal degenerative diseases ${ }^{7,8}$.

hPSCs spontaneously differentiate into RPE cells after removal of basic fibroblast growth factor (bFGF), used to maintain the pluripotency state, from the culture medium ${ }^{9-11}$. The distinctive cobblestone morphology of RPE cells as well as their pigmentation allow to manually collect pigmented areas that appear upon differentiation of hPSCs to obtain a pure population of hPSC-RPE cells. Such approach of RPE cell production is used as cell

${ }^{1}$ INSERM U861, I-Stem, AFM, Institute for Stem cell Therapy and Exploration of Monogenic diseases, 91100, CorbeilEssonnes, France. ${ }^{2}$ UEVE U861, I-Stem, AFM, Institute for Stem cell Therapy and Exploration of Monogenic diseases, 91100, Corbeil-Essonnes, France. ${ }^{3}$ CECS, I-Stem, AFM, Institute for Stem cell Therapy and Exploration of Monogenic diseases, 91100, Corbeil-Essonnes, France. Correspondence and requests for materials should be addressed to C.M. (email: cmonville@istem.fr) 
replacement material in on going and planned clinical trials ${ }^{12-16}$. However, this spontaneous method remains fastidious, inefficient and time consuming ( 8 to 12 weeks of hPSCs differentiation) making it incompatible with the industrial large-scale production which is required to treat the potential millions of patients.

During the last ten years, several teams have developed improved differentiation protocols by combining the use of an increasing number of cytokines and small molecules selected on the basis of results obtained from developmental studies ${ }^{17-20}$. One of the quickest and most efficient protocol was published by Leach et al. in $2015^{20-22}$. Following data demonstrating that RPE and neural retina progenitors (NRPs) have the same embryonic origin, they combined a protocol allowing the efficient differentiation of $\mathrm{NRPs}^{23}$ with previously described RPE inducing factors such as Nicotinamide (NIC) and Activin $\mathrm{A}^{24}$, a member of the TGF- $\beta$ super family. Using this method, they obtained a large majority of cells expressing the pigmentation marker PMEL17 after 14 days of differentiation allowing bypassing manual enrichment of pigmented cells. It is, however, important to note that even with this protocol the banking of mature RPE cells is only performed after several additional weeks of maturation (Day $84)^{22}$.

Therefore, although the differentiation of hPSCs into RPE cells became more efficient during the last years, it still remains a long and laborious process requiring meticulous manipulations from hPSCs thawing to hPSC- RPE cell banking. Many cell culture parameters, such as seeding homogeneity, the time spent by the cells out of the incubator or the method used to isolate pigmented clumps, could impact on the proliferation and the differentiation of hPSCs. Thus, manual processing implies operator to operator variability ${ }^{25,26}$ and the quality of hPSCs and the efficiency of their differentiation into RPE cells are currently highly dependent on technical skills. In this regard, automation should not only allow scaling up the production of hPSC-RPE cells but should also increase its robustness. It could enable larger and more reliable cell production for clinical and disease modeling applications. Robots, such as Cell ${ }^{\text {host }}$ system or CompacT SelecT system, as well as appropriate programing were already developed for the amplification of hPSCs. Differentiation into mesenchymal and neural stem cells has been also implemented in such systems $\mathrm{s}^{27-32}$. Until recently, the requirement of a manual enrichment to obtain a pure population of hPSC-RPE cells prevented the use of these automated systems for the differentiation of this cell type. However, the recent development of protocols allowing efficient differentiation of hPSCs into RPE cells offers now the possibility to automate the production of these cells.

Thus, our aim was to develop a fully automated process allowing the large-scale production of hPSC-RPE cells. Considering that, in addition to considerably complicate the process, the use of numerous growth factors and small molecules on a large scale should be very expensive, especially for an automated process which requires significant dead volumes, we implemented a simplified RPE differentiation protocol amenable for automation that only requires the treatment of hPSCs with NIC, Activin A and CHIR99021, an activator of the Wnt canonical pathway, in a sequential manner. Our protocol recapitulates the main steps of retinal development and is sufficient to obtain a pure population of RPE cells without manual enrichment. We then programmed a culture robot to automate this protocol in order to upscale the production process. 16 billion of mature and functional RPE cells could now be produced within 12 weeks with only one round of production. Such efficient and reproducible automated protocol should be useful for the treatment of the millions of patients affected by RPE associated retinal degeneration.

\section{Results}

Sequential use of nicotinamide, activin A and Chir99021 improves RPE differentiation by recapitulating the main steps of retinal development. In an effort to simplify previous directed differentiation protocols for automation, we evaluated whether the simple use of NIC, Activin A and Chir99021 in a sequential manner (referred as "directed protocol") improves RPE cell differentiation of adherent hESCs enough to bypass manual enrichment. We compared the efficiency of our "directed protocol" with the one of the classical spontaneous differentiation.

We first checked whether the sequential use of NIC, Activin A and Chir99021 could recapitulate the main steps of retinal development by evaluating the expression of markers of the early eye field stage, optic vesicle stage and immature RPE cells at different time points during the differentiation (Fig. 1A). The use of NIC for the first 7 days of differentiation significantly enhanced the transient expression of the early eye field transcription factors SIX homeobox 3 (SIX3) and Retinal homeobox (RAX) concomitantly to a higher decrease of the expression of the pluripotency marker NANOG at mRNA level when compared to the spontaneous protocol ( $\mathrm{p}<0.01 ;$ Fig. 1B). This eye field specification was confirmed at the protein level with the co-expression of the LIM homeobox 2 (LHX2) and the Paired box 6 (PAX6) proteins by most cells at day 7 after NIC treatment $(86.8 \% \pm 4.3 \%, n=3)$, while only $44.3 \%( \pm 2.2 \%, n=3)$ of the non-treated cells express these two markers. Overall, our data suggested that the addition of NIC for 7 days promotes the exit of hESCs from their pluripotent state toward the eye field lineage with a better efficiency than the spontaneous differentiation.

Consecutive treatment with Activin A from day 7 to day 14 significantly increased the expression at mRNA levels of two transcription factors involved in optic vesicle patterning, the visual system homeobox 2 gene (VSX2, also named $C H X 10)$ and the melanocyte inducing transcription factor (MITF), when compared to the spontaneous differentiation (Fig. 1B, $\mathrm{p} \leq 0.05$ ), with an expression peak at day 14 for VSX2. Concomitantly, both $R A X$ and SIX3 mRNA levels were found decreased. Induction of the optic vesicle markers VSX2 and MITF was confirmed by immunofluorescence assays. Cell clusters co-expressing these two proteins were observed by day 10 (Fig. 1D). By contrast on day 14, cells expressing VSX2 were distinct from those expressing MITF, suggesting rapid co-repression of these two genes as described previously (Fig. 1E) ${ }^{33,34}$.

Finally, activation of the canonical WNT signaling pathway by CHIR99021 treatment from day 14 to day 35-42 induced RPE commitment as seen by the acute decreased expression of VSX2 mRNA levels (Fig. 1B) and the continuous increased expression of MITF. MITF expression is significantly upregulated between day 14 and day 30 in the directed protocol when compared to the spontaneous one $(\mathrm{p}<0.01)$. Immunostaining assays 
A.

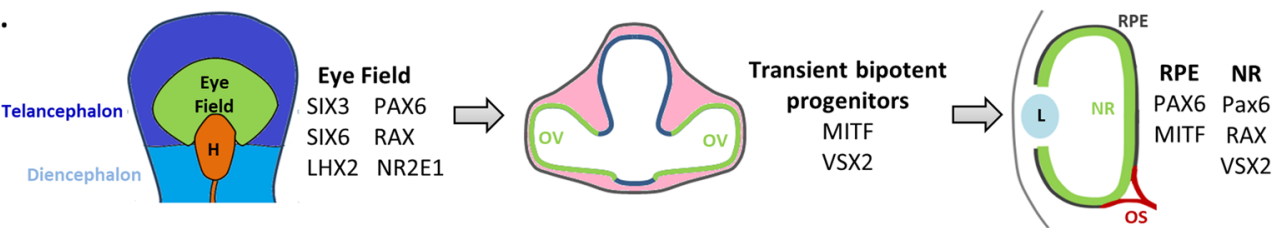

B.
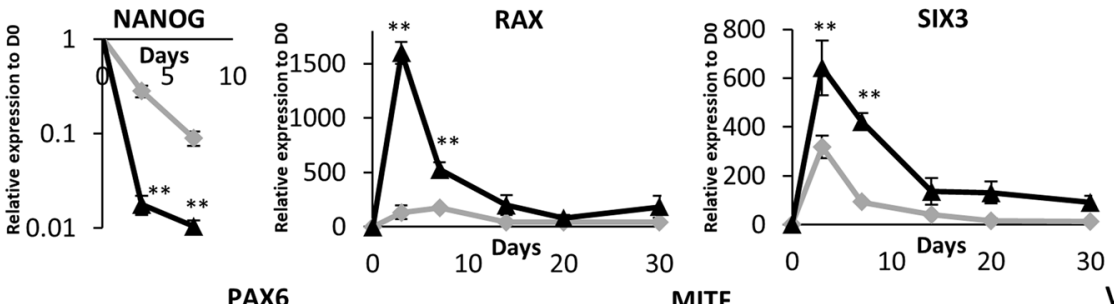

Spontaneous
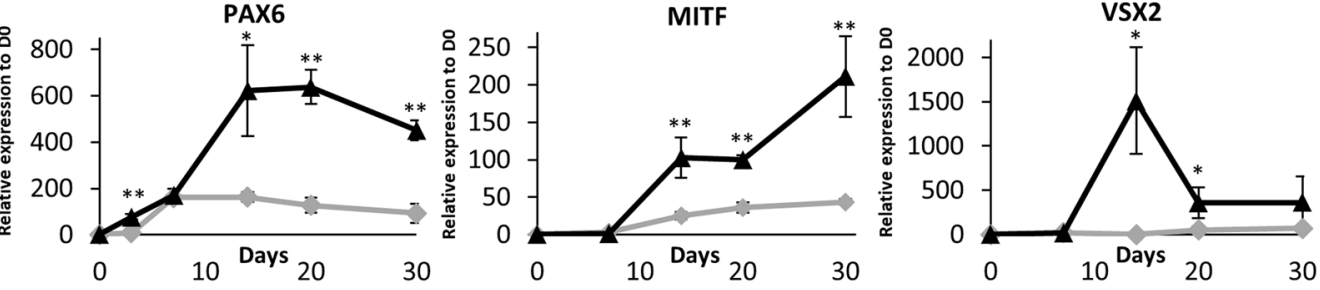

C.

D7

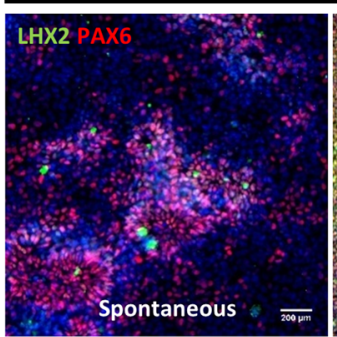

E.

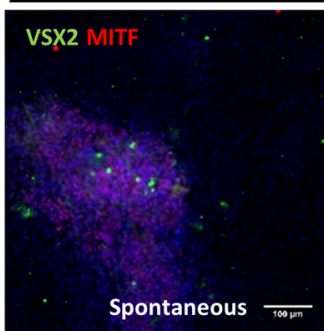

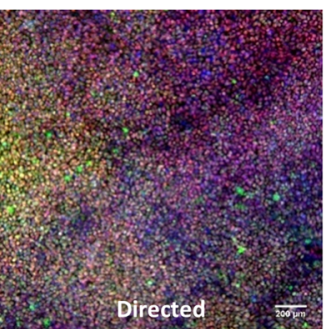

D14

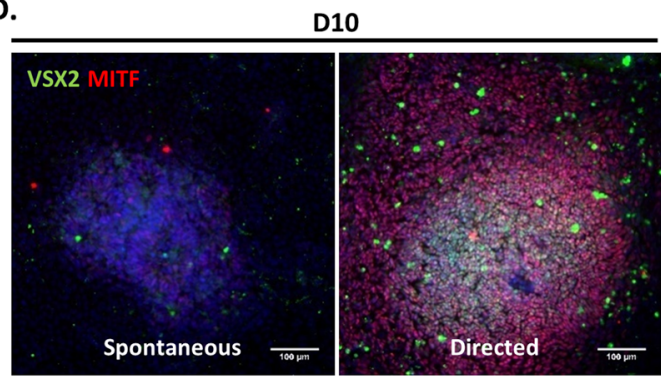

F.
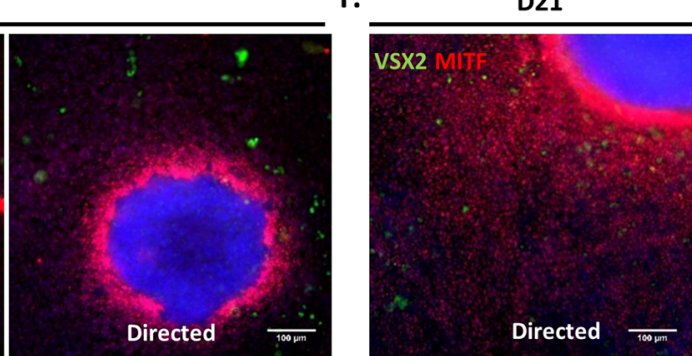

Figure 1. Use of nicotinamide, Activin A and ChiR99021 in a sequential manner recapitulates the main steps of retinal development. (A) Schematic representation of the retinal development. H, Hypothalamus; OV, Optic Vesicle; L, Lens; NR, Neural Retina; RPE, Retinal Pigment Epithelium; OS, Optic Stalk. (B) Relative gene expressions were quantified by RT-qPCR and normalized to mRNA expression at day $0(\mathrm{n}=3$, mean $\pm \mathrm{SD})$. Control condition corresponds to RPE 20\% KSR medium. (C) Representative immunofluorescence for PAX6 and LHX2 at day 7 and for VSX2 and MITF at day 10 (D), D14 (E) and D21 (F). Nuclei are stained with DAPI (blue).

confirmed the absence of VSX2 positive cells at day 21 and the increased number of $\mathrm{MITF}^{+}$cells $(87.5 \% \pm 12.5 \%)$ (Fig. 1F). At this stage putative RPE precursors MITF-positive cells emerged and organized around 3D structures that did not express MITF and VSX2 (Fig. 1F).

We then determined the efficiency of RPE cell induction after 6 weeks of differentiation. A large majority of the culture dish with cells exposed to the directed protocol $(72.96 \% \pm 1.94 \%$ of the culture area, $\mathrm{n}=3)$ was covered by pigmented cells on day 42 (Fig. 2B,C). By contrast, only isolated patches of pigmentation were visible with the spontaneous protocol $(3.481 \% \pm 1.12 \%$ of the growth area, $\mathrm{p}<0.01)$ as reported in a previous study ${ }^{11}$ (Fig. 2B,C). Importantly, the vast majority of cells obtained after 42 days of differentiation with the directed protocol co-expressed PAX6 and MITF $(82.2 \% \pm 3.2 \%, n=3)$, two markers of RPE cells (Fig. 2D).

Taken together our results indicate that the sequential use of Nicotinamide, Activin A and Chir99021 recapitulates the main steps of retinal development and efficiently directs the differentiation of hPSCs into a 
A.
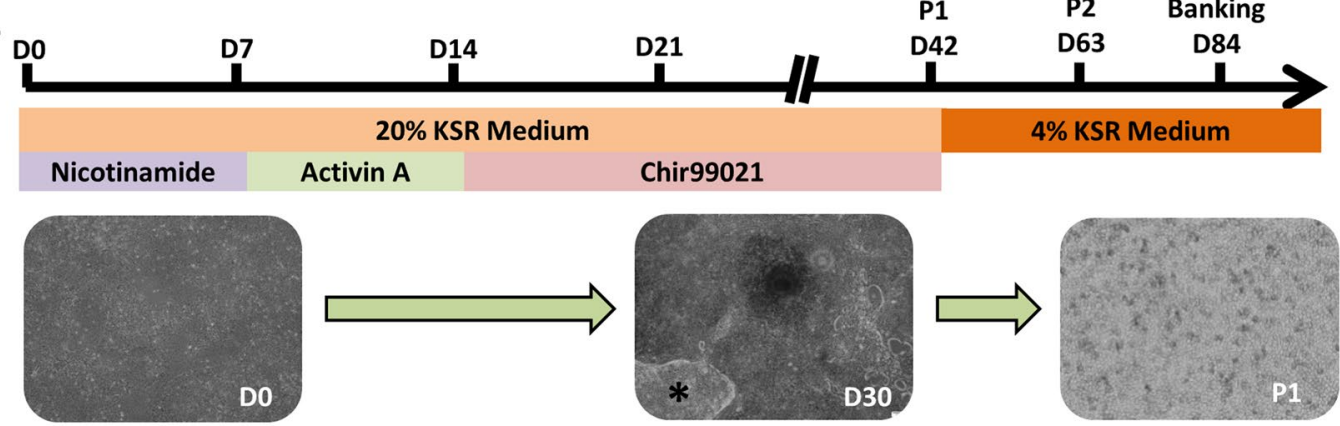

B.

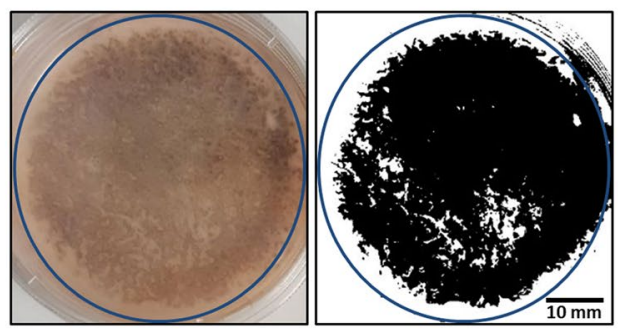

C.
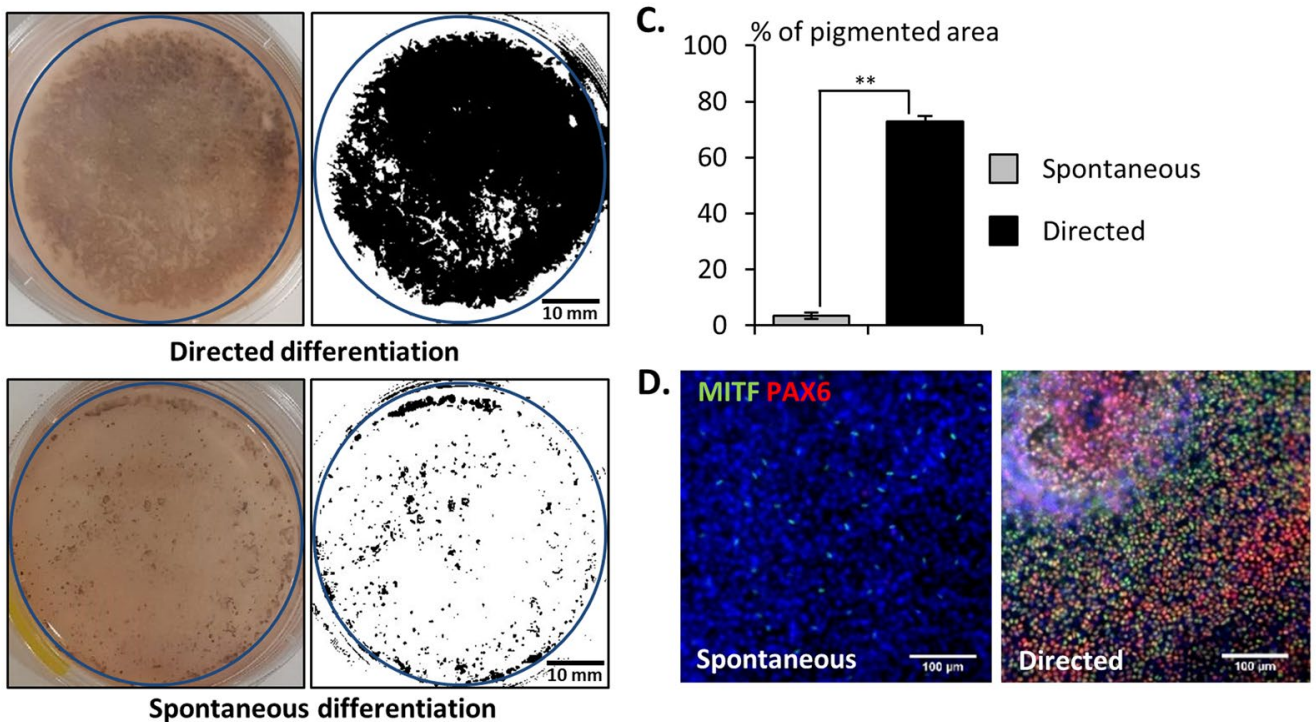

Figure 2. Directed differentiation protocol improves RPE differentiation. (A) Schematic representation of the directed differentiation protocol (black star: cell contaminants). (B) Representative macroscopic observation of the culture dishes after 42 days of differentiation (blue circles: quantified areas) and (C) quantification of the pigmented area $(n=3$, mean $\pm S D)$, (D) Representative immunofluorescence images for the RPE markers PAX6 and MITF after 42 days of differentiation. Nuclei are stained with DAPI (blue).

highly-enriched RPE population within 42 days compared to the spontaneous differentiation. Thus, cell differentiated through the directed protocol could be amplified directly while a prior manual selection of RPE clusters is required for the spontaneous protocol.

The directed protocol allows obtaining a pure population of hESC-RPE cells without manual enrichment and is amenable to automation of the differentiation. Using the "directed differentiation" protocol, we set up a fully automated process by performing media changes and enzymatic passaging using the CompacT SelecT automation platform. This automated cell culture platform is composed of an incubator, bar-coded flasks for cell process tracking, multiple connected pumps to dispense culture media, a six-axis anthropomorphic robotic arm and a live-cell imaging system (Incucyte) (Figs 3 and S2).

Automation starts from the seeding of hPSCs onto $75 \mathrm{~cm}^{2}$ flasks. Then, cell proliferation and differentiation initiation by medium switching were performed in the robot until day 42 . At this stage, hESC-RPE cells form a cohesive epithelium in culture that requires long incubation times with dissociation reagents to trigger cell detachment for further replating and amplification. In order to eliminate a maximum of cell contaminants we took advantage of this characteristic by performing a differential dissociation treatment with TrypLE Express (Fig. 3). We were able to remove the vast majority of unpigmented cells that have lower adherence to the flask than RPE cells on day 42 by applying a first short incubation of 10 minutes with TrypLE Express followed by a rinse. A second enzyme incubation of 35 minutes then enabled the detachment and dissociation of RPE cells.

As the automated system has no centrifugation capability, it was not possible to eliminate the TrypLE Express used to dissociate RPE cells. Thus, we assessed if its dilution in fresh medium after passaging did not affect the re-adherence and growth of the cells. hESC-RPE cells replated with no centrifugation step to remove the dissociation reagent after passaging at day 42 retain their proliferative capacity to form a cohesive epithelium in culture and presented no particular cell death or abnormal morphology (Fig. S3A,B). We also checked by RT-qPCR that the presence of diluted TrypLE Express did not affect RPE identity and once again no difference was detected in RPE gene expression between enzymatic passaging with or without centrifugation (Fig. S3C). 


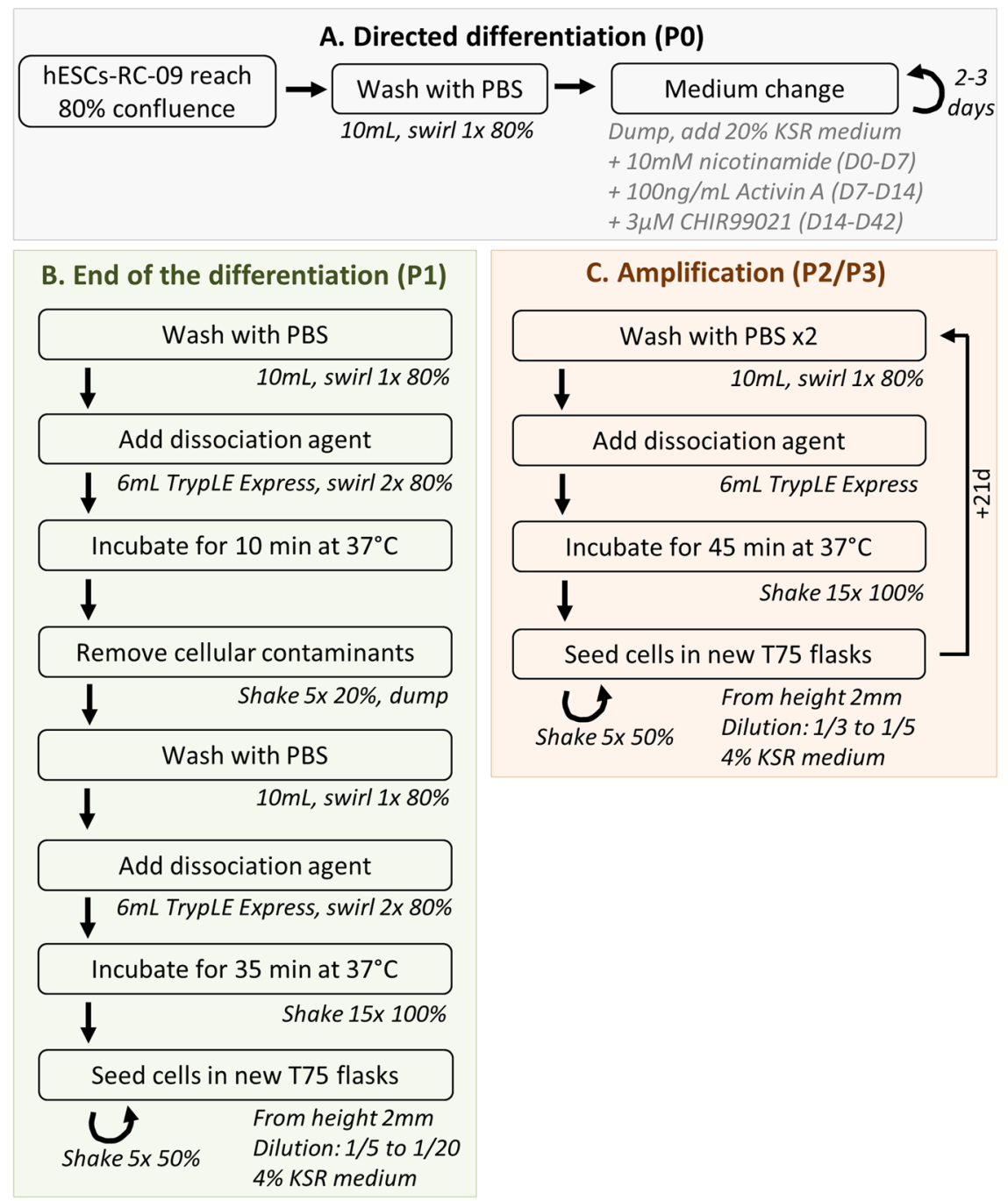

Figure 3. Flowchart of the automated passaging of hESC-RPE cells using the Compact Select automation platform.

After 2 automatized passages, $94.7 \% \pm 0.2 \%(n=3)$ of cells co-expressed the two transcription factors PAX6 and MITF (Fig. 4A) indicating a homogenous population of hESC-RPE cells comparable with the one obtained after manual enrichment ${ }^{13}$. The gene expression of late RPE markers such as RPE65 and CRALBP was also detected by RTqPCR at a level similar to the cells obtained with the manual spontaneous differentiation protocol (Fig. 4B). We further characterized the cell population by flow cytometry and found that $96.8 \% \pm 1.9(\mathrm{n}=3)$ of the cells expressed the pigmentation marker tyrosinase related protein 1 (TYRP1) at passage 2 (Fig. 4C).

All together these data demonstrate that we were able to obtain pure bona fide hESC-RPE cells in an automated system with a quality similar to the cells obtained through the widely used spontaneous differentiation method.

hESC-RPE cells obtained by an automated differentiation are mature and functional. Important issues concerning cells differentiated from hPSCs are their maturity and functionality. As an indicator of epithelial maturity, we evaluated the apico-basal polarization of specific RPE markers. As expected, hESC-RPE cells homogeneously expressed the microvilli protein EZRIN $(95.0 \% \pm 2.8 \%, \mathrm{n}=3)$, the tight junction marker Zonula Occludens-1 $(\mathrm{ZO}-1,99.3 \% \pm 0.4 \%, \mathrm{n}=3)$ and the MER proto-oncogene tyrosine kinase receptor (MERTK, $97.1 \% \pm 1.1 \%, \mathrm{n}=3$ ) at their apical membrane while the calcium activated chloride channel, BESTROPHIN (BEST, $89.4 \% \pm 3.9 \%, \mathrm{n}=3$ ) was localized at the baso-lateral compartment (Figs 5A and S4).

One of the most important functions of RPE cells is the phagocytosis of the outer segments shed by the photoreceptors. To determine whether the cells differentiated according to our directed protocol on the automated cell culture platform were functional, we assessed their ability to phagocyte pig fluorescein isothiocyanate (FITC)labeled photoreceptor outer segments and quantified the fluorescence signal of $\mathrm{pH}$-sensitive particles that become fluorescent after cell entry and phagosome formation. hESC-RPE cells were able to phagocyte FITC-labeled photoreceptor outer segments as shown by the cytoplasmic localization of the FITC signal under the apical limit Ezrin positive (Fig. 5B). hESC-RPE cells incubated with $\mathrm{pH}$-sensitive particles at $37^{\circ} \mathrm{C}$ had a fluorescence 
A.
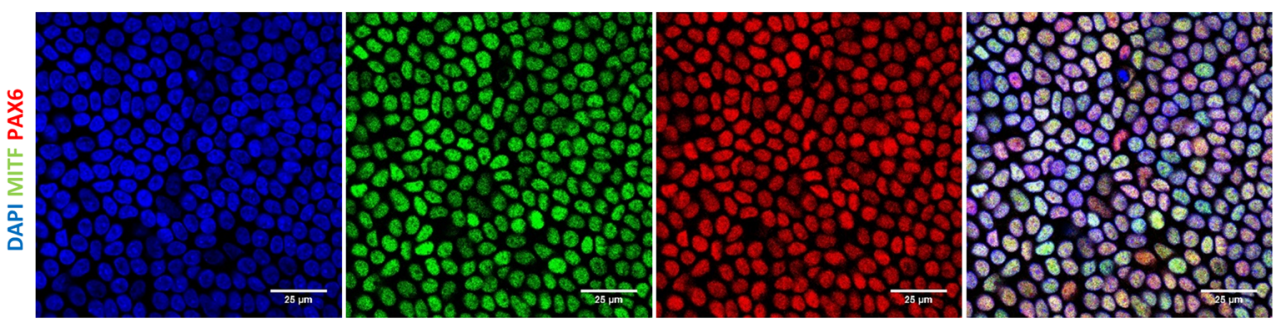

B.

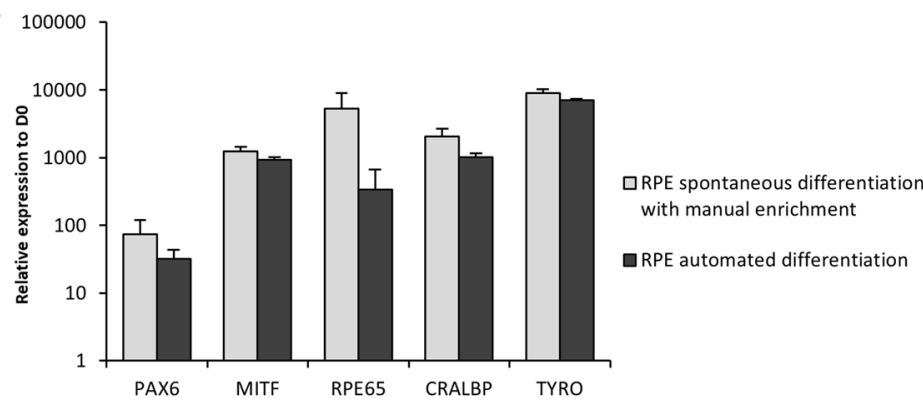

C.

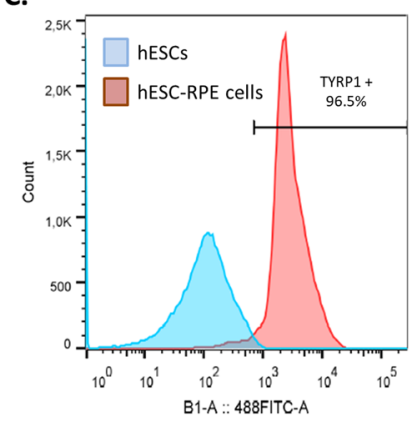

Figure 4. Automated differentiation and amplification of a pure population of hESC-RPE cells without manual selection. (A) Representative immunofluorescence and quantification for the RPE markers MITF and PAX6 at passage 2 after 21 days of culture. Nuclei are stained with DAPI. (B) Relative gene expression of RPE markers quantified by RT-qPCR $(\mathrm{n}=3$, mean \pm SD). (C) Representative flow cytometry histogram for the pigmentation marker TYRP1.

A.
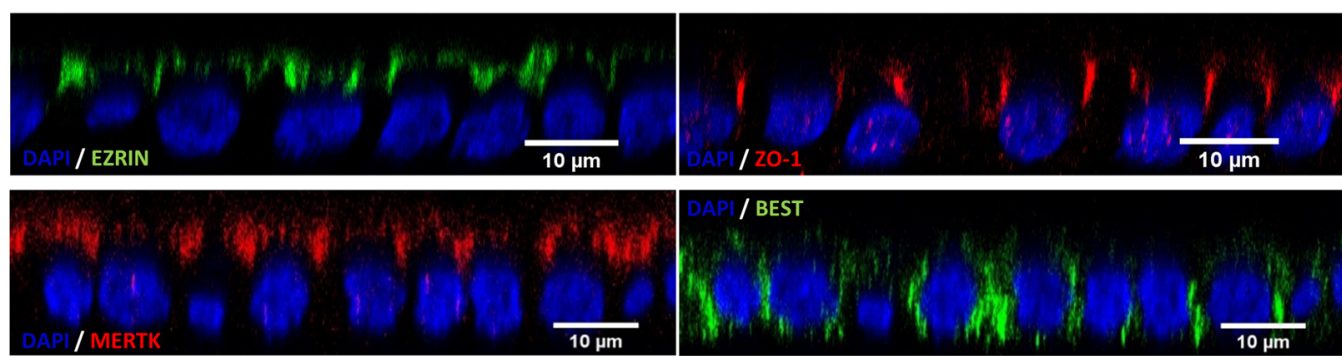

B.
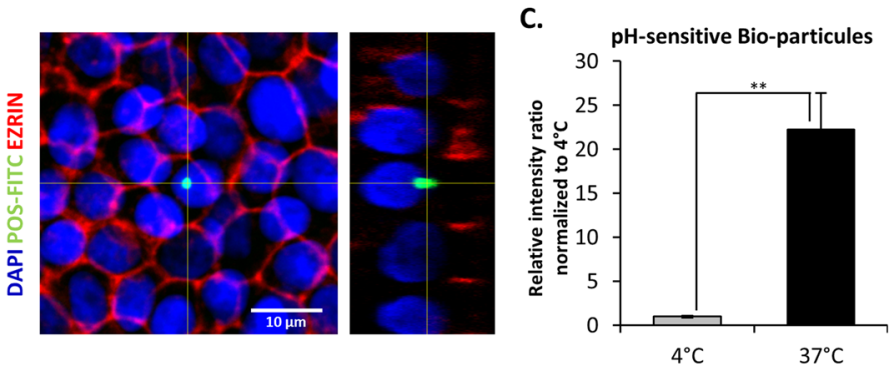

D.

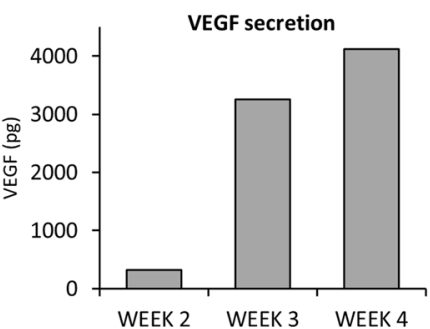

Figure 5. hESC-RPE cells obtained by automated differentiation are mature and functional. (A) Confocal images (projection of XZ planes) for the RPE markers EZRIN, BEST, ZO-1 and MERTK. Nuclei are stained with DAPI. (B) Confocal Images (projection of YZ planes) of hESC-RPE cells after 24 hours of exposure to fluorescein isothiocyanate (FITC)-labeled pig photoreceptor cell outer segments (FITC-POS, green; EZRIN, red; DAPI, blue). (C) Fluorescence intensity quantification of hESC-RPE cells after exposure to $\mathrm{pH}$-sensitive bioparticles $(n=3$, mean $\pm S D)$. (D) Quantification of vascular endothelial growth factor (VEGF) secreted by hESC-RPE cells at different time points using an ELISA assay (Representative assay).

intensity 22.2 fold higher compared to cells incubated at $4{ }^{\circ} \mathrm{C}$, a temperature that inhibits the phagocytic process (Fig. 5C). Another indicator of RPE functionality is the ability to secrete a wide range of growth factors including the vascular endothelial growth factor (VEGF). We quantified the secretion of VEGF after several culture weeks and we observed a progressive increased of VEGF secretion starting from 2 weeks of culture (Fig. 5D). 
A.

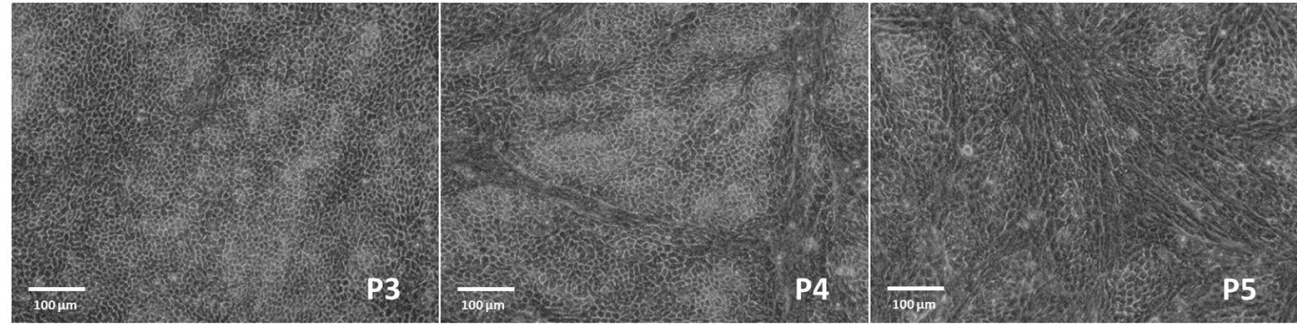

B.
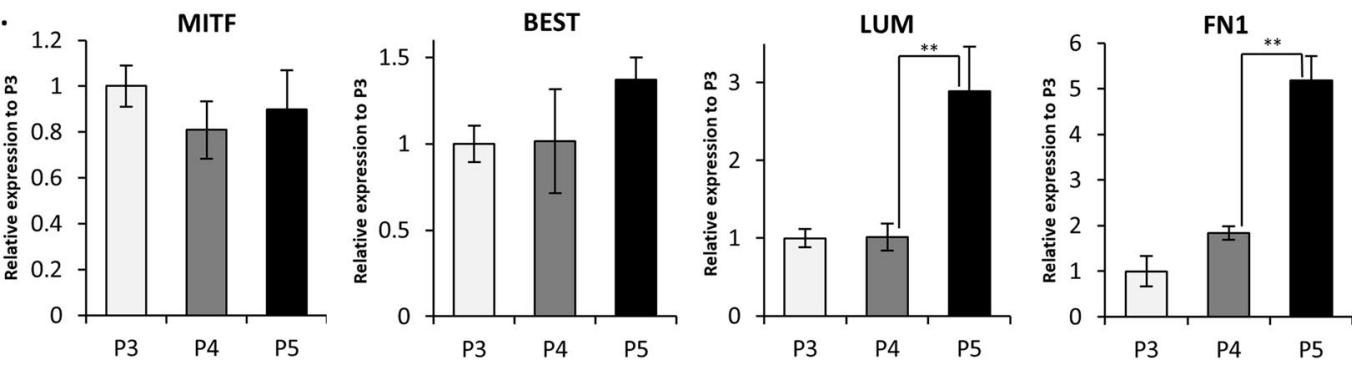

Figure 6. hESC-RPE cells obtained by automated differentiation can be maintained in culture until passage 3 before starting an EMT. (A) Light microscopy images of hESC-RPE cells at passage 3, 4 and 5 at day 21. (B) Relative gene expression of EMT (LUM and FN1) and RPE (MITF and BEST) markers quantified by RT-qPCR $(\mathrm{n}=3$, mean $\pm S D)$.

All these results indicate that RPE cells differentiated from hPSCs using a fully automated protocol are functional in vitro.

hESC-RPE cells differentiated through automation can be amplified until passage 3 to produce large cell banks. Previous studies showed that hESC-RPE cells had a limited amplification potential before they undergo an epithelial-mesenchymal transition (EMT) ${ }^{35,36}$. In line with these studies, hESC-RPE cells obtained with an automated process adopted a mesenchymal phenotype starting from passage 4 (Fig. 6A) despite the maintenance of the gene expression of the RPE markers MITF and BEST (Fig. 6B). Indeed, the cells switched from a classical cobblestone organization to elongated cell morphology (Fig. 6A). This microscopic observation is correlated with the rising expression of mesenchymal markers LUMICAN (LUM) and FIBRONECTIN 1 (FN1), two extracellular matrix proteins, starting at passage 5 when compared to passage 3 and 4 ( $p<0.01$; Fig. 6B). This suggests an EMT transition of hESC-RPE cells, which however maintain an RPE identity. Consequently, we decided to bank our cells at passage 2 using an automated cell banking system (Fill-it, Sartorius) to obtain bona fide hESC-RPE cells at passage 3 after thawing.

\section{Discussion}

Since the publication of the first protocol showing that hESCs spontaneously differentiate into RPE cells, extensive efforts have been made to reduce the duration and increase the efficiency of differentiation protocols ${ }^{17,18,20,24}$. Previous works described that the sequential use of NIC and Activin A allowed increasing embryonic body commitment into retinal lineage. In another hand, Leach et al. showed that the same factors, in addition to many others including bFGF, Noggin, DKK1 (Dickkopf WNT signaling pathway inhibitor 1), Insulin Growth Factor (IGF)-1, Chir99021, B27 and N2 supplements, increased RPE cell differentiation of hPSCs ${ }^{21}$. We demonstrated in this study that most of these cytokines and supplements were not essential to trigger an efficient and pure RPE cell differentiation. Indeed, the use of only 3 compounds (NIC, Activin A and CHIR99021) in a sequential manner allowed obtaining a pure population of RPE cells without 3-dimensional culture and manual dissection of pigmented foci during the differentiation process. This optimized differentiation is thus amenable to automation.

As previously described ${ }^{20,24}$, the use of Nicotinamide quickly reduced the expression of pluripotent markers, suggesting the rapid exit from pluripotent state, while increasing eye field genes expression in treated cells. In this study, Activin A increased both expressions of the neural retina marker VSX2 and the RPE marker MITF. These two markers are initially co-expressed by the same cells at day 10 suggesting that at this stage the cells could be bipotent ${ }^{37}$ and able to give rise to both RPE and neural retina progenitors. But quickly, two subpopulations of cells expressing either one of these two genes appeared. This result is consistent with the known role of VSX2 on MITF repression during optic vesicle patterning ${ }^{33}$. The increased VSX2 expression following Activin A treatment is however surprising as it is known to induce RPE specification rather than neural retina ${ }^{38}$. This result could be due to the endogenous expression of bFGF that positively regulates the expression of VSX2. The simultaneous use of a FGF inhibitor, such as SU5402, already used in a previously described protocol, may be necessary at this stage to repress neural retina markers ${ }^{39}$. The presence of cells expressing only VSX2, meaning that they are potentially engaged into the neural retinal lineage, could be a concern at this stage. However, the later use of the small molecule CHIR99021 rapidly leads to the acute decrease of VSX2 expression concomitantly with an increased expression of MITF, indicating that the activation of the Wnt/ $\beta$-catenin pathway at this stage might induce the 
death of VSX2 positive cells or their conversion into RPE precursors. CHIR99021 treatment could also induce the differentiation of VSX2 positive cells into other mature lineages that are further eliminated by the differential dissociation.

The "directed protocol" developed in this study enabled us to obtain $87.5 \%$ of RPE precursors that expressed MITF by day 21. However, cells were maintained further in culture in order to allow more maturation. Once a cohesive epithelium is formed at day 42 , cells were passaged. This cohesive epithelium is suitable to a differential dissociation that eliminates the last cell contaminants without manual enrichment. Compared with the protocol published by Foltz et al. ${ }^{22}$ where cells are banked at day 84, we bank hESC-RPE cells with the same timing (D84). Thus, the removal of factors other than NIC, Activin A and CHIR99021, used in Foltz and collaborators study did not delay the overall production of hPSC-RPE cells nor affected the quality of the cells obtained.

Using the automated differentiation process described in this study, it is theoretically possible to produce about 16 billion of hESC-RPE cells at passage 2 per batch (Fig. S5). A bank of this size is much larger than those previously described by us and others ${ }^{13,17,40}$ that range from 0.05 to $0.8 \times 10^{9}$ cells, and could be produced by a single operator supervising the robot. Moreover, the use of HYPERflask (Corning) with a growth area of $1720 \mathrm{~cm}^{2}$ (compared to $75 \mathrm{~cm}^{2}$ flask used in this study) could even dramatically increase the number of cells produced per batch. Another way to increase the size of the bank would be to delay the EMT. Indeed, the number of passages without EMT might be extended as previously described by the addition of a ROCK inhibitor in the culture medium $^{35}$.

hPSC-RPE cells have been already grafted in AMD patients either as a cell suspension ${ }^{15,41,42}$ or a polarized epithelium resting on a synthetic basement membrane $e^{14,43}$. The use of cell suspension formulation considerably simplifies the logistical and surgical procedures but several studies made in animal models, including our own, suggest that the survival of the RPE cells and the visual benefits for the animal are improved when the cells are grafted as an epithelial tissue rather than a cell suspension ${ }^{13,44}$. In human, these two approaches have shown both satisfactory safety results and promising efficacy results, even if the extent and the causes of visual improvement in transplant recipients remain ambiguous ${ }^{16}$. Nevertheless, considering that $1 \times 10^{5} \mathrm{hESC}$-RPE cells are currently used to graft a human eye with the both methods ${ }^{14,15,43}$, the automated process presented here should allow to produce enough cells to treat several thousands of patients with retinal degeneration even if some steps of the production, such as the simultaneous banking of a huge numbers of cryovials, remains challenging. The automated culture system could be qualified for clinical cell productions, as actually already done by others ${ }^{45}$. However, before reaching a clinical application, the raw materials used with this automated protocol might be adjusted to comply with Good Manufacturing Practice (GMP) guidelines.

In conclusion, following the previously published amplification of hPSCs using CompacT SelecT automate ${ }^{46}$, we described a fully automated RPE cell differentiation process from the hPSCs thawing to the banking of differentiated cells. Such automated process is a step towards the scale up and the industrialization of RPE differentiation that will be necessary to treat large numbers of patients. Finally, any differentiation protocol that doesn't require $3 \mathrm{D}$ culture or manual selection could theoretically be adapted to this automated culture system opening new perspectives concerning the scale up and the industrialization of the production of many cell types differentiated from hPSCs.

\section{Materials and Methods}

Manual hESCs culture and RPE cell differentiation. Clinical-grade hESC line RC-09 ${ }^{13,47}$ was used and cultured in feeder free conditions using $\mathrm{mTeSR}^{\mathrm{TM}} 1$ Medium (StemCell technologies) and hESC qualified Matrigel (Corning) (Fig. S1). Cells were banked at passage 36 and used for RPE differentiation between passage 38 and 45. Cells were plated at $5 \times 10^{4}$ cells per $\mathrm{cm}^{2}$ and grown until they reached 80 percent of confluence before switching to a differentiation medium composed of Dulbecco's modified Eagle's medium (high glucose, Thermo Fisher Scientific) supplemented with $50 \mu \mathrm{M} \beta$-mercaptoethanol, $1 \times$ minimum essential medium-nonessential amino acids (Thermo Fisher Scientific) and 20\% (D0-D42) or 4\% (after passage 1) of knockout serum replacement (KSR, Thermo Fisher Scientific). During all the differentiation process the medium was changed every $2 / 3$ days.

hESC-RPE cells were obtained by spontaneous differentiation of hESCs as previously described ${ }^{25}$. Briefly, hESCs were grown to confluence and switched to a bFGF deprived culture medium. Pigmented patches were then dissected under a stereomicroscope with a fine $15^{\circ}$ ophthalmic knife and plated onto culture dishes coated with hESC qualified Matrigel (corning).

For the referred "directed differentiation" protocol, $10 \mathrm{mM}$ Nicotinamide (Sigma), $100 \mathrm{ng} / \mathrm{ml}$ Activin A (Peprotech) and $3 \mu \mathrm{M}$ CHIR99021 (Tocris) were added sequentially to the basal differentiation medium at specific time points (Fig. 2A). On day 42, cells were incubated with TrypLE Express Reagent (Thermo Fisher Scientific) for 10 minutes to remove cell contaminants, then washed with PBS and re-incubated with TrypLE Express Reagent for 35 minutes to allow RPE dissociation. Cells were then seeded at a final dilution of 1/5 in dishes coated with hESC qualified Matrigel (Corning).

Mature hESC-RPE cells were dissociated and cryopreserved in liquid nitrogen vapors with CryoStor CS10 medium (StemCell technologies) at passage 1 or 2.

Automated RPE differentiation process. The CompacT SelecT (Sartorius) is a fully automated cell culture platform which allows the expansion and differentiation of large batches of adherent cells in a controlled environment (Fig. S2). The system allows the automation of media changes and cell passaging as well as the monitoring of culture vessels with the automated live-cell imaging system IncuCyte (Sartorius). Contrary to the manual protocol, cells were not centrifuged after dissociation but were directly seeded into new flasks with enough medium to ensure that the final concentration of TrypLE Express reagent in the daughter flasks did not exceed $5 \%$. The automated process is presented in Fig. 3. 
Automated medium changes consisted in (i) retrieving T-flask from the incubator, removing flask cap and pouring media into a waste funnel, (ii) adding the appropriate culture medium using the peristaltic pump system ("dispensing" step), and (iii) replacing T-flask in the flask carousel incubator. Automated passaging consisted in step (i) described above and (ii) add PBS, (iii) swirl flask with the robot arm to ensure an even coverage of PBS across the cell sheet to wash cells, (iv) empty flask, (v) add TrypLE Express, swirl flask and incubate at $37^{\circ} \mathrm{C}$ for $45 \mathrm{~min}$, (vi) add fresh medium to dilute the dissociation reagent, (vii) shake flask from side-to-side at a given speed a number of times to dissociate the cell sheet into single cells or small clumps ("shaking" step), (vii) seed the cell suspension into new T-flasks with a split ratio of 1:3 or 1:5, swirl and replace in the incubator ("seeding" step). Step (v) was modified for the first cell passaging at the end of the differentiation (P0) to perform a two-step enzymatic dissociation procedure to enrich the culture for RPE cells by: (i) incubating the cells for $10 \mathrm{~min}$ with TrypLE Express, (ii) gently shaking the flask and pouring the dissociation agent into a waste funnel to remove non-RPE cells, (iii) incubating the cells for $35 \mathrm{~min}$ with TrypLE Express (“differential dissociation” step).

Quantitative real-time polymerase chain reaction. Total RNAs were extracted using RNAeasy Plus Mini kit (Qiagen) and cDNA synthesized using SuperScript III (Invitrogen). Quantitative real-time RT-PCR was performed using a Quant Studio $12 \mathrm{~K}$ flex (Applied Biosystems) with HiGreen qPCR Master Mix (Thermo Fisher Scientific). Primer sequences are listed in Supplementary Table 1. Experiments were performed with at least three technical replicates per plate and expression levels were normalized to $18 \mathrm{~S}$. Relative expression compared to hESCs gene expression levels were determined by calculating the $2^{-\Delta \Delta \mathrm{Ct}}$.

Immunostaining. hESC-RPE cells were grown on Matrigel-coated 96 or 24-well plates. Adherent cells were fixed in 4\% PFA for $10 \mathrm{~min}$ at room temperature (RT) and rinsed 3 times with PBS. After 30 min in blocking solution (10\% FBS in $0.1 \%$ Triton PBS) at RT, cells were incubated with primary antibodies overnight at $4{ }^{\circ} \mathrm{C}$ (Antibodies are listed in Supplementary Table 2). After 3 washes in PBS, appropriate Alexa Fluor-conjugated secondary antibodies (Invitrogen) were added at 1:500 for $1 \mathrm{~h}$ at RT in presence of DAPI (Invitrogen).

Image acquisition and analysis. Images were acquired with an Axio observer Z1 microscope (Zeiss) with a Hamamatsu ORCA-flash 4.0 camera and a spinning disk unit (Yokogawa CSU-X1-A1N-E; Camera evolve, EMCCD 512) with Metamorph software or with a LSM-800 confocal microscope (Zeiss) with Zen software. Images were exported, analyzed and processed with Fiji software. For zx images, xy stacks ( $0.33 \mu \mathrm{m} \mathrm{z}$ step size) covering cell width were resliced in zx. The quantification of pigmented areas was performed after manual delimitation of culture dish areas using Fiji software. Pictures were then binarized to 8-bit images using a fixed intensity threshold and the black area fraction was measured.

Flow cytometry. Cells were detached from culture plates, fixed in $4 \%$ PFA for $10 \mathrm{~min}$ at RT and permeabilized with PBS containing $0.1 \%$ Triton for $30 \mathrm{~min}$ before labeling with TYRP1 antibody for $1 \mathrm{hr}$ at RT. Labeling of the cell surface markers TRA-1-81 and SSEA4 was performed on freshly dissociated cells for $15 \mathrm{~min}$ at $4{ }^{\circ} \mathrm{C}$. Cells were then incubated with fluorochrome-conjugated primary antibody for $30 \mathrm{~min}$ at RT and rinsed twice with PBS. The antibodies used and their working dilutions are listed in Supplementary Table 2. Cells were analyzed using a cell MACSquant analyzer (MiltenyiBiotec). Gates were drawn according to fluorescence minus one (FMO) controls or on samples labeled with isotype control antibodies. Data were analyzed using FlowJo software (Tree Star, Ashland, OR).

Phagocytosis assay. hESC-RPE cells were exposed for 24 hours to purified FITC-labeled photoreceptor outer segments of pig (gift from Dr. E. Nandrot). After washing with PBS, cells were fixed in cold methanol and labelled with DAPI. Images were taken with LSM-800 confocal microscope (Zeiss). hESCs derived RPE cells were also exposed to pHrodo Green Zymosan Bioparticles (Thermo Fisher Scientific) overnight at $37^{\circ} \mathrm{C}$. These particles are $\mathrm{pH}$-sensitive and become fluorescent after cell entry and phagosome formation. As a negative control, phagocytosis assays were performed at $4{ }^{\circ} \mathrm{C}$ to block the phagocytic process. Plates were then read using a microplate reader (Clariostar-BMG LABTECH) and values were normalized to DAPI intensities.

VEGF quantification by ELISA assay. VEGF measurements were done in triplicate using the human VEGF Quantikine ELISA kit (R\&D System) according to manufacturer instruction.

Statistical analysis. All experiments were performed in triplicate. Summary statistical analyses were performed in XLSTAT software. Comparisons between experiments were performed using the unpaired t-test and statistical significance was established as $* \mathrm{p}<0.05, * * \mathrm{p}<0.01$.

\section{Data Availability}

Additional data and information for reproducing the results described in the article are available from the corresponding author on reasonable request.

\section{References}

1. Strauß, O. Pharmacology of the retinal pigment epithelium, the interface between retina and body system. Eur. J. Pharmacol. 787, 84-93 (2016)

2. Ambati, J. \& Fowler, B. J. Mechanisms of age-related macular degeneration. Neuron 75, 26-39 (2012).

3. Ben M'Barek, K., Habeler, W. \& Monville, C. Stem Cell-Based RPE Therapy for Retinal Diseases: Engineering 3D Tissues Amenable for Regenerative Medicine. Adv. Exp. Med. Biol. 1074, 625-632 (2018). 
4. Wong, W. L. et al. Global prevalence of age-related macular degeneration and disease burden projection for 2020 and 2040: a systematic review and meta-analysis. The Lancet Global Health 2, e106-e116 (2014).

5. Sacchetti, M., Mantelli, F., Merlo, D. \& Lambiase, A. Systematic Review of Randomized Clinical Trials on Safety and Efficacy of Pharmacological and Nonpharmacological Treatments for Retinitis Pigmentosa. Journal of Ophthalmology, https://doi. org/10.1155/2015/737053 (2015).

6. Hernández-Zimbrón, L. F. et al. Age-Related Macular Degeneration: New Paradigms for Treatment and Management of AMD. Oxid Med Cell Longev 2018 (2018).

7. Ramsden, C. M. et al. Stem cells in retinal regeneration: past, present and future. Development 140, 2576-2585 (2013).

8. Forest, D. L., Johnson, L. V. \& Clegg, D. O. Cellular models and therapies for age-related macular degeneration. Dis Model Mech 8, 421-427 (2015).

9. Ferguson, L. R., Balaiya, S., Mynampati, B. K., Sambhav, K. \& Chalam, K. V. Deprivation of bFGF Promotes Spontaneous Differentiation of Human Embryonic Stem Cells into Retinal Pigment Epithelial Cells. J Stem Cells 10, 159-170 (2015).

10. Klimanskaya, I. et al. Derivation and comparative assessment of retinal pigment epithelium from human embryonic stem cells using transcriptomics. Cloning Stem Cells 6, 217-245 (2004).

11. Buchholz, D. E. et al. Derivation of functional retinal pigmented epithelium from induced pluripotent stem cells. Stem Cells 27, 2427-2434 (2009).

12. Bharti, K. et al. Developing cellular therapies for retinal degenerative diseases. Invest. Ophthalmol. Vis. Sci. 55, 1191-1202 (2014).

13. Ben M'Barek, K. et al. Human ESC-derived retinal epithelial cell sheets potentiate rescue of photoreceptor cell loss in rats with retinal degeneration. Sci Transl Med 9 (2017).

14. da Cruz, L. et al. Phase 1 clinical study of an embryonic stem cell-derived retinal pigment epithelium patch in age-related macular degeneration. Nat. Biotechnol. 36, 328-337 (2018).

15. Schwartz, S. D. et al. Embryonic stem cell trials for macular degeneration: a preliminary report. The Lancet 379, 713-720 (2012).

16. Ben M'Barek, K. \& Monville, C. Cell Therapy for Retinal Dystrophies: From Cell Suspension Formulation to Complex Retinal Tissue Bioengineering. Stem Cells Int 2019, 4568979 (2019).

17. Choudhary, P. et al. Directing Differentiation of Pluripotent Stem Cells Toward Retinal Pigment Epithelium Lineage. Stem Cells Transl Med 6, 490-501 (2017).

18. Iwasaki, Y. et al. Differentiation/Purification Protocol for Retinal Pigment Epithelium from Mouse Induced Pluripotent Stem Cells as a Research Tool. PLoS One 11, e0158282 (2016).

19. Lidgerwood, G. E. et al. Defined Medium Conditions for the Induction and Expansion of Human Pluripotent Stem Cell-Derived Retinal Pigment Epithelium. Stem Cell Rev 12, 179-188 (2016).

20. Buchholz, D. E. et al. Rapid and efficient directed differentiation of human pluripotent stem cells into retinal pigmented epithelium. Stem Cells Transl Med 2, 384-393 (2013).

21. Leach, L. L., Buchholz, D. E., Nadar, V. P., Lowenstein, S. E. \& Clegg, D. O. Canonical/3-catenin Wnt pathway activation improves retinal pigmented epithelium derivation from human embryonic stem cells. Invest. Ophthalmol. Vis. Sci. 56, 1002-1013 (2015).

22. Foltz, L. P. \& Clegg, D. O. Rapid, Directed Differentiation of Retinal Pigment Epithelial Cells from Human Embryonic or Induced Pluripotent Stem Cells. J Vis Exp, https://doi.org/10.3791/56274 (2017).

23. Lamba, D. A., Karl, M. O., Ware, C. B. \& Reh, T. A. Efficient generation of retinal progenitor cells from human embryonic stem cells. Proc. Natl. Acad. Sci. USA 103, 12769-12774 (2006).

24. Idelson, M. et al. Directed differentiation of human embryonic stem cells into functional retinal pigment epithelium cells. Cell Stem Cell 5, 396-408 (2009).

25. Lane, A. et al. Engineering Efficient Retinal Pigment Epithelium Differentiation From Human Pluripotent Stem Cells. Stem Cells Transl Med 3, 1295-1304 (2014).

26. Veraitch, F. S., Scott, R., Wong, J.-W., Lye, G. J. \& Mason, C. The impact of manual processing on the expansion and directed differentiation of embryonic stem cells. Biotechnol. Bioeng. 99, 1216-1229 (2008).

27. Paull, D. et al. Automated, high-throughput derivation, characterization and differentiation of induced pluripotent stem cells. Nat. Methods 12, 885-892 (2015).

28. Jenkins, M. J. \& Farid, S. S. Human pluripotent stem cell-derived products: advances towards robust, scalable and cost-effective manufacturing strategies. Biotechnol J 10, 83-95 (2015).

29. Terstegge, S. et al. Automated maintenance of embryonic stem cell cultures. Biotechnol. Bioeng. 96, 195-201 (2007).

30. Konagaya, S., Ando, T., Yamauchi, T., Suemori, H. \& Iwata, H. Long-term maintenance of human induced pluripotent stem cells by automated cell culture system. Sci Rep 5, 16647 (2015).

31. Thomas, R. J. et al. Automated, scalable culture of human embryonic stem cells in feeder-free conditions. Biotechnol. Bioeng. 102, 1636-1644 (2009).

32. Terstegge, S., Pochert, J. \& Brüstle, O. Hamilton's new cellhost system for full automation of embryonic stem cell cultures. Nature Methods | Application Notes 271-272, https://doi.org/10.1038/nmeth1204-271 (2004).

33. Horsford, D. J. et al. Chx10 repression of Mitf is required for the maintenance of mammalian neuroretinal identity. Development 132, 177-187 (2005)

34. Wang, Z., Yasugi, S. \& Ishii, Y. Chx10 functions as a regulator of molecular pathways controlling the regional identity in the primordial retina. Developmental Biology 413, 104-111 (2016).

35. Croze, R. H. et al. ROCK Inhibition Extends Passage of Pluripotent Stem Cell-Derived Retinal Pigmented Epithelium. Stem Cells Transl Med 3, 1066-1078 (2014)

36. Singh, R. et al. Functional Analysis of Serially Expanded Human iPS Cell-Derived RPE Cultures. Invest Ophthalmol Vis Sci 54, 6767-6778 (2013).

37. Zhong, X. et al. Generation of three dimensional retinal tissue with functional photoreceptors from human iPSCs. Nat Commun $\mathbf{5}$, 4047 (2014).

38. Fuhrmann, S., Levine, E. M. \& Reh, T. A. Extraocular mesenchyme patterns the optic vesicle during early eye development in the embryonic chick. Development 127, 4599-4609 (2000).

39. Meyer, J. S. et al. Modeling early retinal development with human embryonic and induced pluripotent stem cells. Proc Natl Acad Sci USA 106, 16698-16703 (2009).

40. Lu, B. et al. Long-Term Safety and Function of RPE from Human Embryonic Stem Cells in Preclinical Models of Macular Degeneration. Stem Cells 27, 2126-2135 (2009).

41. Schwartz, S. D. et al. Human embryonic stem cell-derived retinal pigment epithelium in patients with age-related macular degeneration and Stargardt's macular dystrophy: follow-up of two open-label phase 1/2 studies. The Lancet 385, 509-516 (2015).

42. Schwartz, S. D., Tan, G., Hosseini, H. \& Nagiel, A. Subretinal Transplantation of Embryonic Stem Cell-Derived Retinal Pigment Epithelium for the Treatment of Macular Degeneration: An Assessment at 4 Years. Invest. Ophthalmol. Vis. Sci. 57, ORSFc1-9 (2016).

43. Kashani, A. H. et al. A bioengineered retinal pigment epithelial monolayer for advanced, dry age-related macular degeneration. Science Translational Medicine 10, eaao4097 (2018).

44. Diniz, B. et al. Subretinal implantation of retinal pigment epithelial cells derived from human embryonic stem cells: improved survival when implanted as a monolayer. Invest. Ophthalmol. Vis. Sci. 54, 5087-5096 (2013). 
45. Hourd, P. et al. Qualification of academic facilities for small-scale automated manufacture of autologous cell-based products. Regen Med 9, 799-815 (2014).

46. Archibald, P. R. T. et al. Comparability of automated human induced pluripotent stem cell culture: a pilot study. Bioprocess Biosyst Eng 39, 1847-1858 (2016).

47. De Sousa, P. A. et al. Derivation of the clinical grade human embryonic stem cell line RCe013-A (RC-9). Stem Cell Res 17, 36-41 (2016).

\section{Acknowledgements}

We thank E. Nandrot for the gift of FITC-labeled photoreceptor cell outer segment preparations from pigs. We thank Roslin Cells for providing RC-09 hESCs. This work was supported by grants from LABEX REVIVE [ANR-10-LABX-73]. It was supported by NeurATRIS, a translational research infrastructure (Investissements d'Avenir) for biotherapies in Neurosciences [ANR-11-INBS-0011] and INGESTEM, the national infrastructure (Investissements d'Avenir) engineering for pluripotent and differentiated stem cells [ANR-11-INBS-000]. I-Stem is part of the Biotherapies Institute for Rare Diseases supported by the Association Française contre les Myopathies (AFM)-Téléthon.

\section{Author Contributions}

F.R. and A.P. performed manual cell culture and manual RPE cell differentiation experiments. L.L. and L.M. performed automated RPE cell differentiation. F.R., L.L., W.H. and K.B.M. performed RPE quality control evaluations. F.R., K.B.M., L.M. and C.M. discussed the data. F.R., K.B.M. and C.M. wrote the manuscript. All authors reviewed the manuscripts.

\section{Additional Information}

Supplementary information accompanies this paper at https://doi.org/10.1038/s41598-019-47123-6.

Competing Interests: The authors declare no competing interests.

Publisher's note: Springer Nature remains neutral with regard to jurisdictional claims in published maps and institutional affiliations.

(c) (i) Open Access This article is licensed under a Creative Commons Attribution 4.0 International (c) License, which permits use, sharing, adaptation, distribution and reproduction in any medium or format, as long as you give appropriate credit to the original author(s) and the source, provide a link to the Creative Commons license, and indicate if changes were made. The images or other third party material in this article are included in the article's Creative Commons license, unless indicated otherwise in a credit line to the material. If material is not included in the article's Creative Commons license and your intended use is not permitted by statutory regulation or exceeds the permitted use, you will need to obtain permission directly from the copyright holder. To view a copy of this license, visit http://creativecommons.org/licenses/by/4.0/.

(c) The Author(s) 2019 